\title{
The flatness theorem for non-symmetric convex bodies via the local theory of Banach spaces
}

\author{
Wojciech Banaszczyk (Łódź) \\ Alexander E. Litvak (Tel Aviv) \\ Alain Pajor (Marne-la-Vallée) \\ Stanislaw J. Szarek (Cleveland \& Paris)
}

\begin{abstract}
Let $L$ be a lattice in $\mathbb{R}^{n}$ and $K$ a convex body disjoint from $L$. The classical Flatness Theorem asserts that then $\mathrm{w}(K, L)$, the $L$-width of $K$, doesn't exceed some bound, depending only on the dimension $n$; this fact was later found relevant to questions in integer programming. Kannan and Lovász (1988) showed that under the above assumptions $\mathrm{w}(K, L) \leq C n^{2}$, where $C$ is a universal constant. Banaszczyk (1996) proved that $\mathrm{w}(K, L) \leq C n(1+\log n)$ if $K$ has a centre of symmetry. In the present paper we show that $\mathrm{w}(K, L) \leq C n^{3 / 2}$ for an arbitrary $K$. It is conjectured that the exponent $3 / 2$ may be replaced by 1 , perhaps at the cost of a logarithmic factor; we prove that for some naturally arising classes of bodies.
\end{abstract}

Keywords: convex bodies, lattices, flatness theorem, Gaussian measure 


\section{Introduction}

The study of convex sets is central in many areas of mathematics. One of these is optimization, be it discrete or continuous. Another, at the first sight unrelated, is functional analysis: balls in normed spaces are convex and, conversely, a "non-degenerated" convex symmetric set in a vector (or affine) space induces a norm for which it is a ball (symmetry is an important but not a crucial restriction). This article employs methods developed and/or commonly used in the latter field to investigate the questions in convexity motivated by the former.

"When does a convex set contain a point with integer coordinates?" is, in essence, the Integer Programming Problem. Lenstra (1983) showed that, in fixed dimension, the problem can be solved in polynomial time, and the argument involves the notion of lattice width of a set $K$, defined (for the integer lattice $\mathrm{Z}^{n}$ ) by

$$
\mathrm{w}\left(K, \mathrm{Z}^{n}\right):=\min \left\{\max _{x \in K}\langle x, y\rangle-\min _{x \in K}\langle x, y\rangle: y \in \mathbb{Z}^{n} \backslash\{0\}\right\},
$$

where $\langle x, y\rangle$ is the canonical inner product in $\mathbb{R}^{n}$. If $K \subset \mathbb{R}^{n}$ is a convex body that is integer point free (i.e., $K \cap \mathrm{Z}^{n}=\emptyset$ ), then $\mathrm{w}\left(K, \mathrm{Z}^{n}\right)$ is bounded by some constant, depending only on the dimension $n$; this fact, due to Khinchine (1948), is usually called the flatness theorem. The value of that constant is relevant to the complexity of Lenstra's algorithm. Given a convex body $K \subset \mathbb{R}^{n}$ denote

$\operatorname{Flt}(K):=\sup \left\{\mathrm{w}\left(H, Z^{n}\right): H\right.$ is an affine image of $K$ satisfying $\left.H \cap \mathbb{Z}^{n}=\emptyset\right\}$.

Kannan and Lovász (1988) proved that if $K$ is an $n$-dimensional convex body, then $\operatorname{Flt}(K) \leq C n^{2}$ (here and below, $C, c, c_{0}, c^{\prime}$ etc. denote universal numerical constant which may vary between occurrences). We refer to their paper for details and more exhaustive motivational and bibliographical information concerning the flatness theorem. It was later proved by Banaszczyk (1996) that $\operatorname{Flt}(K) \leq C n(1+\log n)$ if $K$ is symmetric, and that $\operatorname{Flt}(K) \leq n$ if $K$ is an ellipsoid. On the other hand, one has $\operatorname{Flt}(K) \geq n$ if $K$ is a simplex, and the results of Banaszczyk (1996) imply that $\operatorname{Flt}(K) \geq c n$ for all symmetric $K$ (cf. Proposition 2.3 below).

The present paper was motivated by an attempt to improve the upper bound for $\operatorname{Flt}(K)$ for non-symmetric $K$. We show here (Theorem 2.4, Corollary 2.5) that $\operatorname{Flt}(K) \leq C_{0} n^{3 / 2}$ for an arbitrary $n$-dimensional $K$, and that 
$\operatorname{Flt}(K) \leq C_{1} n(1+\log n)$ if $K$ is a simplex or, more generally, a polytope whose number of vertices or faces is polynomial in the dimension (the constant $C_{1}$ depending then on the polynomial in question), which is the principal case of interest if $K$ is defined by its faces or vertices. Then we use these results to improve the inequalities given in Kannan and Lovász (1988) and estimating $\mathrm{w}(K, L)$ by the number of points of $L$ in $K$ (see Remark 2.7).

Let us sketch the ideas involved in the arguments. If $K \subset \mathbb{R}^{n}$ is a convex body containing the origin in the interior, the polar body $K^{\circ}$ is defined by

$$
K^{\circ}:=\left\{x \in \mathbb{R}^{n}:\langle x, y\rangle \leq 1 \text { for each } y \in K\right\} .
$$

One has $\left(K^{\circ}\right)^{\circ}=K$. We denote further by $\|\cdot\|_{K}$ the gauge function of $K$ (i.e., $\|x\|_{K}:=\inf \{t>0: x / t \in K\}$ ) and set

$$
M(K):=\int_{S^{n-1}}\|x\|_{K} d \mu_{n}(x)
$$

where $\mu_{n}$ is the normalized Lebesgue measure on $S^{n-1}$. The quantity $M(K)$ is equal to one half of a well-known geometric parameter: the mean width of $K^{\circ}$.

We show first that $\operatorname{Flt}(K) \leq C n M(K) M\left(K^{\circ}\right)$; this inequality is actually quite deep as it relies on the Majorizing Measure Theorem for Gaussian processes (Talagrand (1987)) - why Gaussian, it will become apparent in the sequel. Since $\operatorname{Flt}(K)$ is an affine invariant of $K$, the problem is to find an affine image $H$ of $K$ with $M(H) M\left(H^{\circ}\right)$ as small as possible. If $K$ is symmetric and if $X=\left(\mathbb{R}^{n},\|\cdot\|_{K}\right)$ is the corresponding normed space, then it is a known fact in the local theory of Banach spaces that one can find $H$ with

$$
M(H) M\left(H^{\circ}\right) \leq K(X) \leq C(1+\log n)
$$

where $K(X)$ is the so-called $K$-convexity constant of $X$ (see e.g., Lindenstrauss and Milman (1993), (4.1.9) and (4.1.10), or Pisier (1989), Theorem 3.1; we give $a$ definition in section 4 ). This is sometimes referred to as the " $M M^{*}$-estimate" and asserts that, as far as the " $M M^{*}$-product" is concerned, an arbitrary $n$-dimensional normed space differs from the Euclidean space $l_{2}^{n}$ (in which case the product clearly equals 1 ) only by a logarithmic factor. In the geometric language this means that every (bounded symmetric) convex body admits an affine image so "well balanced" that its mean width and simultaneously that of its polar are "essentially" the same as is the case for the Euclidean ball. This is quite surprising in view of the fact that 
the Banach-Mazur distance (see section 4 for definition) of an $n$-dimensional normed space to $l_{2}^{n}$ could be as large as $\sqrt{n}$ (resp. $n$ in the non-symmetric case), and that, moreover, for many - perhaps most - of "standard" classes of spaces there are universal (i.e., independent of the space and its dimension) estimates for the $K$-convexity constant.

The statements about the parameter $M(\cdot)$ (and, particularly, the " $M M^{*}$ product") are often expressed in the closely related language of Gaussian rather than spherical averages. Let $\gamma_{n}$ be the standard Gaussian measure on $\mathbb{R}^{n}$ with density $(2 \pi)^{-n / 2} e^{-|x|^{2} / 2}$, where $|\cdot|$ is the usual Euclidean norm. If $K \subset \mathbb{R}^{n}$ is a convex body containing the origin in the interior, let us denote

$$
\ell(K):=\left(\int_{\mathbb{R}^{n}}\|x\|_{K}^{2} d \gamma_{n}(x)\right)^{1 / 2}
$$

If $K$ is 0 -symmetric, then $\ell(K)$ is just the so-called $\ell$-norm of the identity operator acting from the Euclidean space $\mathbb{R}^{n}$ to the normed space $X=$ $\left(\mathbb{R}^{n},\|\cdot\|_{K}\right)$; see e.g., Lindenstrauss and Milman (1993), (2.3.16) and (2.3.17). The Gaussian average $\ell(K)$ is "essentially" the same as $n^{1 / 2} M(K)$. More precisely, there are constants $\sigma_{n} \geq 1$ with $\sigma_{n} \rightarrow 1$ as $n \rightarrow \infty$ such that, for all $K$ as above, $\ell(K)=\sigma_{n} n^{1 / 2} M(K)$. (We could have dispensed with $\sigma_{n}$ 's if we had defined $M(K)$ via the second moment of $\|\cdot\|_{K}$, an insignificant modification by Lemma 3.3; however, that would not conform to the standard terminology.) Thus, if $K$ is symmetric and $H, X$ are as above, one has $\ell(H) \ell\left(H^{\circ}\right) \leq n K(X) \leq C n(1+\log n)$ while, on the other hand, $\operatorname{Flt}(K)=$ $\operatorname{Flt}(H) \leq C \ell(H) \ell\left(H^{\circ}\right)$ etc.

It is natural to attempt to carry over the scheme described above to the non-symmetric case; unfortunately, no efficient route to that goal is known. Indeed, a straightforward "translation" of the argument allows one only to produce an affine image $H$ of $K$ with $\ell(H) \ell\left(H^{\circ}\right) \leq n^{2}$, which yields an estimate for $\operatorname{Flt}(K)$ of the same order as that obtained in Kannan and Lovász (1988). We show two ways, corresponding to the two parts of Theorem 2.4, to improve that direct approach. The first argument uses the John's decomposition of identity (or, alternatively, a reverse Brascamp-Lieb inequality; see Remark 3.6) to analyse the quantities $\ell(H) \ell\left(H^{\circ}\right)$ directly. In full generality it implies only that $\operatorname{Flt}(K) \leq C n^{3 / 2}(1+\log n)^{1 / 2}$, but allows for superior estimates for polytopes with "not too many" vertices or faces. The second argument adapts the definition of the $K$-convexity constant to the non-symmetric case and uses "non-symmetric Gaussian projections" to yield an estimate of the type $C n^{3 / 2}$. 
We wish to point out here that estimating the product $\ell(H) \ell\left(H^{\circ}\right)$ is (essentially) equivalent to finding the smallest $\lambda$ for which there are $a, b>0$, with $a b \leq \lambda$ such that, say,

$$
\gamma_{n}(a H) \cdot \gamma_{n}\left(b H^{\circ}\right) \geq .8
$$

This can be thought of as a Gaussian analogue of the reverse Santaló inequality (cf. Bourgain and Milman (1987)). In the symmetric case the asymptotically optimal value of $\lambda$ that works for some affine image $H$ of an arbitrary $K$ is somewhere between $O\left(n(1+\log n)^{1 / 2}\right)$ (see Proposition 5.1 in section 5) and $O(n(1+\log n))$ (cf. Bourgain (1984), Pisier (1981); see the comments at the beginning of section 4$)$. In the non-symmetric case the game is wide open; our upper estimate being $O\left(n^{3 / 2}\right)$ and the $n$-dimensional simplex requiring $O(n(1+\log n))$ (again, see Proposition 5.1), which we conjecture is the correct order.

In view of Lemma 2.2 below, we could reduce the non-symmetric case to the symmetric one if we were able to satisfactorily answer the following question: what is the smallest $\lambda>0$ such that for any convex body $K \subset \mathbb{R}^{n}$ with $\gamma_{n}(K-K) \geq .9$ there exists $a \in K$ such that $\gamma_{n}(\lambda(K-a)) \geq .9$ ? Again, we conjecture that the $n$-dimensional regular simplex gives the correct order $O(1+\log n)$ (or perhaps is even optimal).

Since, as indicated earlier, the questions considered here are motivated partly by the Integer Programming Problem, it is appropriate to ask whether the arguments are "constructive", e.g., given a convex body $K$ disjoint from $\mathbb{Z}^{n}$, are we able to "explicitly" exhibit a $y \in \mathbb{Z}^{n} \backslash\{0\}$ that witnesses the "flatness" of $K$ ? We have not pursued this question vigorously; however, at least at first sight, such "constructivity" appears to be difficult to extract from our approach. (The same question arises with $\mathrm{Z}^{n}$ replaced by an arbitrary lattice.)

Our results (both preliminary and main) and their corollaries are listed in section 2. The following two sections deal respectively with the details of the two arguments referred to above. Finally, section 5 addresses mostly the issue of the necessity for logarithmic factors that appear in many places throughout the paper.

After this paper has been submitted, it has been noticed by Litvak and Tomczak-Jaegermann (1998) that our Proposition 3.1 (see also Remark 3.2 implies the following estimate on the Banach-Mazur distance between arbitrary $n$-dimensional not-necessarily-symmetric convex bodies $K_{1}, K_{2}$ (see 
section 4 for definitions)

$$
d\left(K_{1}, K_{2}\right) \leq O\left(n^{3 / 2}(1+\log n)^{1 / 2}\right) .
$$

It has been known for some time that such results can be obtained from " $\ell \ell^{*}-$ " (or " $M M^{*} *_{-}$) estimates (see, e.g., Benyamini and Gordon (1981), Bourgain and Milman (1986)), but in the present context the argument requires some finesse. We should point out here that, until recently, no upper estimate for $d\left(K_{1}, K_{2}\right)$ better than $n^{2}$ (and following from John (1948)) was known; on the other hand, to our knowledge and besides very low dimensions, no examples are known with $d\left(K_{1}, K_{2}\right)>n$ - and certainly none with $d\left(K_{1}, K_{2}\right) \gg n$. Finally, very recently, stronger results were obtained by Rudelson (1998), who proved $O\left(n^{4 / 3}(1+\log n)^{a}\right)$ type estimates $(a>0$ a numerical constant) for both the Banach-Mazur distance and for the " $\ell \ell^{*}$ product". This yields an identical improvement to the first inequality of our Theorem 2.4 and its consequences, including the resulting estimate of $\operatorname{Flt}(K)$ (cf. Proposition 2.3).

Acknowledgement. The first named author was partially supported by KBN Grant 2 P301 019 04; part of the work was done when he was visiting University of Marne-la-Vallée in the spring of 1995 . The research of the second and fourth named authors was partially supported by Grant No.9200285 from U.S.-Israel Binational Science Foundation. The research of the fourth named author was partially supported by NSF (U.S.A.). The second named author would like to thank Professor E. Gluskin for his help and encouragement.

\section{More notation and the results}

Before proceeding further, we need to establish some notation. By $\mathcal{K}^{n}$ we denote the family of all closed bounded convex bodies in $\mathbb{R}^{n}$. It is convenient to introduce the following subfamilies of $\mathcal{K}^{n}$

$$
\begin{aligned}
& \mathcal{K}_{0}^{n}:=\left\{K \in \mathcal{K}^{n}: K \text { contains zero in the interior }\right\}, \\
& \mathcal{C}^{n}:=\left\{K \in \mathcal{K}^{n}: K \text { has a centre of symmetry }\right\} \\
& \mathcal{C}_{0}^{n}:=\left\{K \in \mathcal{K}^{n}: K \text { is symmetric with respect to zero }\right\} .
\end{aligned}
$$

As usual, most of the results can be carried over to the class of closed convex bodies (i.e., possibly unbounded, or containing 0 not necessarily in the 
interior, ...) just by approximation. However, we shall in principle restrict ourselves to the smaller classes $\mathcal{K}^{n}$ (resp. $\mathcal{K}_{0}^{n}$ ) to avoid technical difficulties, even though often, for the purpose of brevity, we shall talk of polars of bodies that could a priori contain zero on the boundary.

Since the invariants we study are (largely) affinely invariant, it will be convenient to consider, in addition to $\mathbb{Z}^{n}$, arbitrary lattices. A lattice in $\mathbb{R}^{n}$ is an additive subgroup of $\mathbb{R}^{n}$ generated by $n$ linearly independent vectors. The family of all lattices in $\mathbb{R}^{n}$ is denoted by $\mathcal{L}^{n}$. Given a lattice $L$, we define the dual lattice $L^{*}$ in the usual way

$$
L^{*}:=\left\{x \in \mathbb{R}^{n}:\langle x, y\rangle \in \mathrm{Z} \text { for each } y \in L\right\}
$$

one has $\left(L^{*}\right)^{*}=L$. The concept of lattice width generalizes; for $K \in \mathcal{K}^{n}$ and $L \in \mathcal{L}^{n}$, the $L$-width of $K$ is

$$
\mathrm{w}(K, L):=\min _{y \in L^{*} \backslash\{0\}}\left\{\max _{x \in K}\langle x, y\rangle-\min _{x \in K}\langle x, y\rangle\right\} .
$$

and it is readily seen that, for $K \in \mathcal{K}^{n}$,

$$
\operatorname{Flt}(K)=\max \left\{\mathrm{w}(K, L): L \in \mathcal{L}^{n}, a \in \mathbb{R}^{n} \text { and } K \cap(a+L)=\emptyset\right\} .
$$

Let $L \in \mathcal{L}^{n}$ and $K \in \mathcal{K}^{n}$. By $\mu(K, L)$ one denotes the covering radius of $L$ with respect to $K$

$$
\mu(K, L):=\min \left\{r>0: L+r K=\mathbb{R}^{n}\right\} .
$$

If $K \in \mathcal{C}_{0}^{n}$, one denotes by $\lambda_{1}(K, L)$ the first minimum of $L$ with respect to $K$

$$
\lambda_{1}(K, L):=\min \left\{\|x\|_{K}: x \in L \backslash\{0\}\right\} .
$$

The lemma that follows connects these two parameters with Gaussian moments of gauges of the convex bodies in question. Before stating it, we wish to comment that although $\ell(K)$ is traditionally defined as the second Gaussian moment, it is occasionally more convenient to work with other moments, say $\ell_{p}(K):=\left(\int_{\mathbb{R}^{n}}\|x\|_{K}^{p} d \gamma_{n}(x)\right)^{1 / p}$ for $p \in(0, \infty)$. We point out that, for a fixed $p, \ell_{p}(K)$ is uniformly equivalent to $\ell(K)$ over $K \in \mathcal{K}_{0}^{n}$, $n \in \mathbb{N}$. This is well known for $K \in \mathcal{C}_{0}^{n}$ (in fact for a much more general class of measures). We provide a proof for the non-symmetric case in Lemma 3.3. The argument gives the correct order of the constants involved as $p \rightarrow+\infty$, but we will use primarily the case $p=1$. 
Lemma 2.1 There exists a universal constant $C_{0}$ such that

$$
\mu(K, L) \cdot \lambda_{1}\left(H, L^{*}\right) \leq C_{0} \ell(K) \ell(H)
$$

for arbitrary $K \in \mathcal{K}_{0}^{n}, H \in \mathcal{C}_{0}^{n}$ and $L \in \mathcal{L}^{n}$.

For $K \in \mathcal{C}_{0}^{n}$, this is just a special case of Lemma 3 of Banaszczyk (1996). The proof given by Banaszczyk (1996), based on the theorem of Talagrand (1987) on majorizing measure, extends unchanged to arbitrary $K \in \mathcal{K}_{0}^{n}$.

Lemma 2.2 For an arbitrary convex body $K \in \mathcal{K}_{0}^{n}$ and $p \in[1, \infty)$, one has

$$
\ell_{p}\left((K-K)^{\circ}\right) \leq 2 \ell_{p}\left(K^{\circ}\right)
$$

with equality for $p=1$.

Proof. For $K, H \in \mathcal{K}_{0}^{n}$ we have

$$
\|\cdot\|_{(K+H)^{\circ}}=\|\cdot\|_{K^{\circ}}+\|\cdot\|_{H^{\circ}},
$$

in particular

$$
\|x\|_{(K-K)^{\circ}}=\|x\|_{K^{\circ}}+\|-x\|_{K^{\circ}} .
$$

The conclusion of the Lemma follows now from the definition of $\ell_{p}(\cdot)$ and the triangle inequality for an $L_{p}$-norm.

Let $K, H \in \mathcal{K}^{n}$. If there is an affine transformation of $\mathbb{R}^{n}$ which carries $K$ onto $H$, then we write $K \sim H$. For $K \in \mathcal{K}^{n}$, we denote

$$
\operatorname{Ell}(K):=\min \left\{\ell(H) \ell\left(H^{\circ}\right): H \in \mathcal{K}_{0}^{n} \text { and } H \sim K\right\} .
$$

It is implicit in the above definition that the bodies $H$ contain 0 in the interior. However, in the sequel we will often suppress for the purpose of brevity, the "interior" requirement in related definitions and statements. We point out that, in any case, the infimum such as above can not be achieved if 0 belongs to the boundary of $H$.

We can now state the following two results, which summarize the present paper. 
Proposition 2.3 There exist universal positive constants $c, C$ such that

$$
c n \leq \operatorname{Flt}(K) \leq C \operatorname{Ell}(K)
$$

for $K \in \mathcal{K}^{n}$.

Proof. The existence of $c>0$ such that $c n \leq \operatorname{Flt}(K)$ for every $K \in \mathcal{C}_{0}^{n}$ is a direct consequence of Theorem 2 of Banaszczyk (1996); the proof extends almost unchanged to arbitrary $K \in \mathcal{K}^{n}$.

For the second inequality, fix $K \in \mathcal{K}^{n}$ and consider an arbitrary $H \in \mathcal{K}_{0}^{n}$ with $H \sim K$. Next, take an arbitrary $L \in \mathcal{L}^{n}$ and $a \in \mathbb{R}^{n}$ with $(L+a) \cap H=$ $\emptyset$; then $\mu(H, L)>1$. It follows directly from the definitions that

$$
\mathrm{w}(H, L)=\lambda_{1}\left((H-H)^{\circ}, L^{*}\right) .
$$

Thus, by Lemmas 2.1 and 2.2, we have

$$
\mathrm{w}(H, L) \leq C_{0} \ell(H) \ell\left((H-H)^{\circ}\right) \leq 2 C_{0} \ell(H) \ell\left(H^{\circ}\right)
$$

where $C_{0}$ is the constant from Lemma 2.1. Since $L$ and $a,(L+a) \cap H=\emptyset$, were arbitrary, it follows that

$$
\operatorname{Flt}(H) \leq 2 C_{0} \ell(H) \ell\left(H^{\circ}\right) .
$$

As $H \sim K$, we have $\operatorname{Flt}(H)=\operatorname{Flt}(K)$. Thus

$$
\operatorname{Flt}(K) \leq 2 C_{0} \ell(H) \ell\left(H^{\circ}\right) .
$$

Since $H \sim K$ was arbitrary, we obtain $\operatorname{Flt}(K) \leq 2 C_{0} \operatorname{Ell}(K)$.

Theorem 2.4 There exists a universal constant $C$ such that

$$
\operatorname{Ell}(K) \leq C n^{3 / 2}, \quad K \in \mathcal{K}^{n} .
$$

Moreover, if $K$ is a polytope with $N_{1}$ vertices and $N_{2}$ faces, then

$$
\operatorname{Ell}(K) \leq C n(1+\log n)^{1 / 2}\left(1+\log \min \left\{N_{1}, N_{2}\right\}\right)^{1 / 2} .
$$

The proof of Theorem 2.4 will be the object of the next two sections. 
Corollary 2.5 Let $S$ be an $n$-dimensional simplex in $\mathbb{R}^{n}$. Then

$$
n \leq \operatorname{Flt}(S) \leq C n(1+\log n),
$$

where $C$ is a universal constant.

Proof. The first inequality is well known: consider the simplex $S$ with vertices $0, n e_{1}, \ldots, n e_{n}$; it is enough to observe that the interior of $S$ is disjoint from $\mathrm{Z}^{n}$ while $\mathrm{w}\left(S, \mathrm{Z}^{n}\right)=n$. The second inequality follows from Proposition 2.3 and the second part of Theorem 2.4.

Remark 2.6 Let us say that a polytope with integer vertices is latticefree if it intersects the integer lattice only at its vertices. Very recently, Kantor (1998) showed that for any positive number $\alpha<\frac{1}{e}$, and for $n$ large enough, there exists a lattice-free simplex $S \subset \mathbb{R}^{n}$ of dimension $n$ whose width $\mathrm{w}\left(S, \mathrm{Z}^{n}\right)>\alpha n$. It can also be shown that $\alpha n$ may be replaced by $n-2$ (Sebö (1998)).

Remark 2.7 Let $K \in \mathcal{K}^{n}, L \in \mathcal{L}^{n}$ and let $s$ be the number of elements of $L$ in $K$. Kannan and Lovász (1988) proved that

$$
\mathrm{w}(K, L) \leq C n^{2}\left\lceil(s+1)^{1 / n}\right\rceil
$$

where $C$ is a universal constant. For $K \in \mathcal{C}^{n}$ they gave the bound

$$
\mathrm{w}(K, L) \leq C_{0} n\left(n+s^{1 / n}\right),
$$

which was improved by Banaszczyk (1996) to

$$
\mathrm{w}(K, L) \leq C_{1} n\left(1+\log n+s^{1 / n}\right) .
$$

(see also Gritzmann and Wills (1993), (3.12) and (3.13)). Talata (1995) improved $(*)$ to

$$
\mathrm{w}(K, L) \leq C^{\prime} n\left(n+s^{1 / n}\right) .
$$

The argument used in Talata (1995) together with the first part of Theorem 2.4 allows one to show that

$$
\mathrm{w}(K, L) \leq C^{\prime \prime} n\left(n^{1 / 2}+s^{1 / n}\right),
$$

while combined with Corollary 2.5 yields

$$
\mathrm{w}(S, L) \leq C_{2} n\left(1+\log n+s^{1 / n}\right)
$$

if $S$ is an $n$-dimensional simplex, with analogous statements if the number of facets or the number of vertices of the body in question is "under control". 


\section{The $\ell \ell^{*}$-estimate via the John's decomposi- tion of identity.}

The object of this section is to prove the following result, which will imply the second part of Theorem 2.4.

Proposition 3.1 There exists a positive constant $C$ such that for any integer $n$ and any $n$-dimensional convex polytope $K$, we have

$$
\operatorname{Ell}(K) \leq C n(1+\log n)^{1 / 2}(1+\log N)^{1 / 2},
$$

where $N$ is the smaller of the number of facets and the number of vertices of $K$.

Remark 3.2 Proposition 3.1 implies, for an arbitrary $K \in \mathcal{K}^{n}$, an estimate

$$
\operatorname{Ell}(K) \leq C_{0} n^{3 / 2}(1+\log n)^{1 / 2}
$$

for some universal constant $C_{0}$. This follows e.g., from the well-known fact that any n-dimensional convex body can be "reasonably" approximated by a polytope with at most, say, $5^{n}$ vertices (or faces). The same estimate follows also directly from the proof of Proposition 3.1. The proof shows that the above estimate on $\operatorname{Ell}(K)$ is witnessed by affine images of $K$ whose Löwner or John ellipsoids are Euclidean balls, see below for definitions (specifically, the estimate given by Proposition 3.1 - and the second part of Theorem 2.4 is witnessed by the affine image of $K$ whose Löwner ellipsoid is a Euclidean ball if the number of faces of $K$ exceeds that of vertices, resp. the John ellipsoid if the opposite is true). We will improve this estimate in the next section by removing the factor $(1+\log n)^{1 / 2}$.

Before proving Proposition 3.1, we observe that the moments of the gauge of a convex body are equivalent. This fact is well known when we the body is symmetric and the measure is log-concave, see for instance Milman and Schechtman (1986), Appendix II.

Lemma 3.3 There is a positive constant c such that for any integer $n$, any $p \geq 1$ and any $K \in \mathcal{K}_{0}^{n}$, we have

$$
\frac{c}{\sqrt{p}} \ell_{p}(K) \leq \int_{\mathbb{R}^{n}}\|x\|_{K} d \gamma_{n}(x) \equiv \ell_{1}(K) .
$$


Proof. By homogeneity, we may assume that $\int_{\mathbb{R}^{n}}\|x\|_{K} d \gamma_{n}(x)=1$. Then, by Markov inequality, $\gamma_{n}(t K)>1-1 / t$, for any $t>0$. In particular, choose $t$ to satisfy $\gamma_{1}((-\infty, 1])=1-1 / t$. Then $t K \supset B_{2}^{n}$ (the Euclidean unit ball). Indeed, if $x \in \mathbb{R}^{n},|x|=1$, was such that $x \notin t K$ then, by a separation argument, there would exist a unit vector $u \in \mathbb{R}^{n}$ verifying

$$
t K \subset\left\{y \in \mathbb{R}^{n}:\langle y, u\rangle \leq\langle x, u\rangle\right\} \subset\left\{y \in \mathbb{R}^{n}:\langle y, u\rangle \leq 1\right\},
$$

whence $\gamma_{n}(t K) \leq \gamma_{1}((-\infty, 1])=1-1 / t$, contradicting our choice of $t$. As a consequence, the gauge of $K$ is $t$-Lipschitz on the Euclidean space. We now use the following variant of the Gaussian isoperimetric inequality (see Pisier (1986)): if $F: \mathbb{R}^{n} \rightarrow \mathbb{R}$ is is $t$-Lipschitz (with respect to the Euclidean metric), then, for any $s>0$,

$$
\gamma_{n}\left(F-\int_{\mathbf{R}^{n}} F d \gamma_{n} \geq s\right) \leq \exp \left(-s^{2} / 2 t^{2}\right)
$$

(any other version of the isoperimetric inequality would also work). Accordingly, $\gamma_{n}\left(\|\cdot\|_{K}-1 \geq s\right) \leq \exp \left(-s^{2} / 2 t^{2}\right)$ and so

$\ell_{p}(K)^{p}=\int_{0}^{+\infty} p z^{p-1} \gamma_{n}\left(\|x\|_{K} \geq z\right) d z \leq 1+\int_{1}^{+\infty} p z^{p-1} \exp \left(-\frac{(z-1)^{2}}{2 t^{2}}\right) d z$.

The right-hand term can easily be estimated from above by $\left(\frac{\sqrt{p}}{c}\right)^{p}$ for some universal constant $c>0$.

The Löwner ellipsoid (resp. John ellipsoid) of a body $K$ is the (unique) ellipsoid of minimal volume containing $K$ (resp. maximal volume contained in $K)$. For any body $K \subset \mathbb{R}^{n}$, there exists an affine transformation $T$ such that the unit Euclidean ball $B_{2}^{n}$ is the Löwner (resp. John) ellipsoid of $T(K)$. An important and useful feature of these concepts is described by the following result of John (1948).

Lemma 3.4 Let $K$ be a body of $\mathbb{R}^{n}$ such that the ellipsoid of minimal volume containing $K$ is the unit Euclidean ball. Then there exist $M$ contact points $\left(u_{i}\right)_{1 \leq i \leq M}\left(\left\|u_{i}\right\|_{K}=\left|u_{i}\right|=1\right.$ for all $\left.1 \leq i \leq M\right)$ and $M$ positive scalars $\left(\lambda_{i}\right)_{1 \leq i \leq M}$ such that

$$
\sum_{i=1}^{M} \lambda_{i}=n, \sum_{i=1}^{M} \lambda_{i} u_{i}=0 \text { and } \forall x \in \mathbb{R}^{n}, x=\sum_{i=1}^{M} \lambda_{i}\left\langle x, u_{i}\right\rangle u_{i} .
$$

Moreover $M$ can be chosen so that $M \leq \frac{n(n+3)}{2}$. 
We will now estimate $\operatorname{Ell}(K)$ using the Löwner ellipsoid of $K$.

Proof of Proposition 3.1. Replacing, if necessary, $K$ by its affine image, we may assume that the Euclidean unit ball is the Löwner ellipsoid of $K$. Let $\left(u_{i}\right)_{1 \leq i \leq M}$ and $\left(\lambda_{i}\right)_{1 \leq i \leq M}$ be as in Lemma 3.4. Then, for any $x \in \mathbb{R}^{n}$, we have

$$
\|x\|_{K} \leq \inf \left\{\sum_{i=1}^{M} t_{i}: x=\sum_{i=1}^{M} t_{i} u_{i}, t_{i} \geq 0, i=1, \ldots, M\right\} .
$$

Since $\sum_{1 \leq i \leq M} \lambda_{i} u_{i}=0$, we have

$$
x=\sum_{i=1}^{M} \lambda_{i}\left\langle x, u_{i}\right\rangle u_{i}=\sum_{i=1}^{M} \lambda_{i}\left(\left\langle x, u_{i}\right\rangle-\min _{1 \leq j \leq M}\left\langle x, u_{j}\right\rangle\right) u_{i} .
$$

Therefore

$$
\|x\|_{K} \leq \sum_{i=1}^{M} \lambda_{i}\left\langle x, u_{i}\right\rangle-n \min _{1 \leq j \leq M}\left\langle x, u_{j}\right\rangle .
$$

We integrate now this inequality over $\mathbb{R}^{n}$. The first term on the right yields zero and so, using Lemma 3.3, we get

$$
\ell(K) \leq c^{-1} \ell_{1}(K) \leq c^{-1} n \int_{\mathbb{R}^{n}} \max _{1 \leq j \leq M}\left\langle x, u_{j}\right\rangle d \gamma_{n}(x)
$$

Estimates of the last integral are well known (see e.g., Pisier (1989), Lemma 4.14), and are stated here as a lemma for future reference.

Lemma 3.5 Let $Z_{1}, \ldots, Z_{M}$ be a sequence of mean zero Gaussian random variables on a probability space $(\Omega, P)$. Then

$$
\int_{\Omega} \max _{1 \leq j \leq M}\left|Z_{j}\right| d P \leq C_{1}(1+\log M)^{1 / 2} \max _{1 \leq j \leq M}\left\|Z_{j}\right\|_{L_{2}(\Omega)}
$$

where $C_{1}$ is a universal constant.

Since in our setting $M \leq n(n+3) / 2 \leq 2 n^{2}$ and since, for $u \in \mathbb{R}^{n}$, the Gaussian variable $Z=\langle\cdot, u\rangle$ is of norm $|u|$ in $L_{2}\left(\mathbb{R}^{n}, \gamma_{n}\right)$, Lemma 3.5 implies

$$
\ell(K) \leq C_{0} n(1+\log n)^{1 / 2} .
$$

On the other hand, the dual norm, the gauge of $K^{\circ}$, is dominated by the Euclidean norm and so

$$
\ell\left(K^{\circ}\right) \leq \ell\left(B_{2}^{n}\right)=\sqrt{n} .
$$


The last two inequalities yield the estimate

$$
\operatorname{Ell}(K) \leq C_{0} n^{3 / 2}(1+\log n)^{1 / 2}
$$

given in Remark 3.2. To obtain the estimate stated in the assertion of Proposition 3.1, let $K$ be a polytope with $N_{1}$ vertices. Then, again by Lemma 3.5,

$$
\ell\left(K^{\circ}\right) \leq C_{1}\left(1+\log N_{1}\right)^{1 / 2} .
$$

Combining this with the estimate for $\ell(K)$ we get that

$$
\operatorname{Ell}(K) \leq C n(1+\log n)^{1 / 2}\left(1+\log N_{1}\right)^{1 / 2} .
$$

On the other hand, if the number of facets of $K$ is $N_{2}$, then so is the number of vertices of $K^{\circ}$, which shows that the inequality above also holds with $N_{1}$ replaced by $N_{2}$. This completes the proof of Proposition 3.1.

Remark 3.6 Very recently Barthe (1998a) showed that among all convex bodies $K$ whose John ellipsoid is the unit Euclidean ball (hence $\ell(K) \leq \sqrt{n}$ ), the mean width $2 \int\|x\|_{K^{\circ}} d \mu_{n}(x)$, is maximal if $K$ is a regular simplex $S$ circumscribed to the unit Euclidean ball; $\mu_{n}$ denotes here the normalized Lebesgue measure on $S^{n-1}$. The argument uses a reverse Brascamp-Lieb inequality (see Barthe (1998b), cf. Brascamp and Lieb (1976)). This implies the same assertion for the Gaussian average. An easy computation shows that for $S, \ell\left(S^{\circ}\right)$ is of order $n(1+\log n)^{1 / 2}$, which, for general $K$, gives the same estimate for $\operatorname{Ell}(K)$ as the one obtained above.

\section{The modified Gaussian projection and the $\ell \ell^{*}$-estimate.}

The purpose of this section is to prove the first part of Theorem 2.4 (i.e., to remove the logarithmic factor from the estimate given in Remark 3.2).

As indicated in the introduction, the situation is quite well understood in the symmetric case. We recall that for two normed spaces $E_{1}, E_{2}$ the Banach-Mazur distance is defined by

$$
d\left(E_{1}, E_{2}\right):=\inf \left\{\|T\| \cdot\left\|T^{-1}\right\|: T: E_{1} \rightarrow E_{2} \text { a linear isomorphism }\right\} .
$$


(As usual in such context we identify a normed space $E$ with its unit ball $B_{E}$, thus we may write $d\left(K_{1}, K_{2}\right)$ in place of $d\left(E_{1}, E_{2}\right)$ if $K_{j}=B_{E_{j}}, j=1,2$.) Then, if $K \in \mathcal{C}_{0}^{n}, X=\left(\mathbb{R}^{n},\|\cdot\|_{K}\right)$ is the corresponding normed space and $d_{X}:=d\left(l_{2}^{n}, X\right) \leq \sqrt{n}$, we have

$\operatorname{Ell}(K) \equiv \min \left\{\ell(u K) \ell\left((u K)^{\circ}\right): u \in G L(n)\right\} \leq C n\left(1+\log d_{X}\right) \leq C n(1+\log n)$,

where $C$ is a universal constant (Figiel and Tomczak-Jaegermann (1979), Lewis (1979), Pisier (1981)). This estimate has many consequences in the finite dimensional geometry.

Closely connected to $\ell \ell^{*}$-estimates is another invariant of a normed space: the norm of the Gaussian projection $\mathrm{G}$ in the space $L^{2}(X)$; that norm can be taken as a definition of $K(X)$, the $K$-convexity constant of $X$, mentioned in the introduction. Let us recall the notion of the Gaussian projection (for details see Pisier (1989), Ch.2). Consider the probability space $(\Omega, \mathcal{A}, P)$ $=\left(\mathbb{R}^{N}, \mathcal{B}, \gamma_{\infty}\right)$, where $\mathcal{B}$ is the Borel $\sigma$-algebra and $\gamma_{\infty}$ is the canonical Gaussian probability measure on $\mathbb{R}^{N}$. Then the coordinate functions $g_{i}$ : $\mathbb{R}^{N} \rightarrow \mathbb{R}$, defined by $g_{i}(x)=x_{i}$, form a sequence of independent identically distributed standard Gaussian random variables.

Let $A$ be the set of all finitely supported sequences $\alpha=\left(\alpha_{1}, \alpha_{2}, \ldots\right)$ with $\alpha_{n} \in \mathrm{Z}^{+}$. For $\alpha \in A$ define

$$
H_{\alpha}(x):=h_{\alpha_{1}}\left(x_{1}\right) h_{\alpha_{2}}\left(x_{2}\right) \ldots,
$$

where $h_{k}(x), k=0,1,2, \ldots$ are Hermite polynomials (recall $h_{0} \equiv 1$ ). Then $\left\{H_{\alpha}: \alpha \in A\right\}$ is an orthogonal basis of $L_{2}=L_{2}(\Omega, P)$.

Let $\mathcal{H}_{k}$ be a closed span in $L_{2}$ of the functions $\left\{H_{\alpha}:|\alpha| \equiv \sum \alpha_{i}=k\right\}$ and $Q_{k}: L_{2} \rightarrow \mathcal{H}_{k}$ be the orthogonal projection. E.g., $Q_{0} f=\int_{\Omega} f d P$ and $Q_{1}$ is the orthogonal projection onto the linear span of $\left\{g_{i}: i \geq 1\right\}$.

As usual, $L_{2}(X)$ denotes the space of measurable functions $f: \Omega \rightarrow X$ for which

$$
\|f\|_{L_{2}(X)}:=\left(\int_{\Omega}\|f(x)\|^{2} d P\right)^{1 / 2}
$$

is finite. As in our setting $\operatorname{dim} X<\infty, L_{2}(X)$ may be identified with $L_{2} \otimes X$ (in general, it would be a completion of $L_{2} \otimes X$ with respect to the above norm). We now define the Gaussian projection $G=G_{X}: L_{2}(X) \rightarrow L_{2}(X)$ by the formula $G=Q_{1} \otimes I_{X}$, where $I_{X}$ denotes the identity operator on $X$. In 
the sequel we will suppress the implicit dependence of $G$ on $X$; in particular we will sometimes write $G$ for $Q_{1}=Q_{1} \otimes I_{\mathbb{R}}=G_{\mathbb{R}}$.

The connection between $\ell \ell^{*}$-estimates and the Gaussian projection is given by the following inequality (Figiel and Tomczak-Jaegermann (1979))

$$
\operatorname{Ell}(K) \leq C_{0}\|G\|
$$

where $K=B_{X}$ and $C_{0}$ is a universal constant. In the Hilbert space case the Gaussian projection is orthogonal, hence of norm one. Surprisingly, that norm is also "quite small" in the case of a general normed space. Indeed, Pisier (1989) (cf. Bourgain (1984)) did show that

$$
\|G\| \leq C_{1}\left(1+\log d_{X}\right)
$$

where $C_{1}$ is a universal constant; this is in general much smaller than the "trivial" estimate $d_{X}$ we could otherwise expect. The last two inequalities clearly imply the estimate for $\operatorname{Ell}(K)$ in the symmetric case stated at the beginning of this section.

As indicated in the introduction, transferring these results to the nonsymmetric setting (which is the natural one from the point of view of convex bodies) is not automatic. Indeed, even the correct statement of the problem encounters difficulties. This is basically due to the fact that the natural context for non-symmetric convex bodies being that of an affine space, the center of the coordinate system is not canonically determined. In accordance with this philosophy we shall consider, as the primary setup, the convex body $K$ as a subset of an $n$-dimensional affine space $V$. Once we decide to choose $a \in V$ (normally an interior point of $K$ ) as the "origin", $V$ may be identified with its underlying vector space $X$ via $v \longrightarrow v-a$, and $K$ with $K-a:=K_{a} \subset X$. We can now consider the gauge of of $K_{a}$ in $X$ (or $V, a$ being fixed). In order to make the notation more transparent we shall denote the gauge function by $p_{K, a}(x)$ if $x$ is thought of as an element of $V$ and by $\|x\|_{K_{a}}$ if $x \in X$. Thus, for $x \in V$,

$$
p_{K, a}(x):=\|x-a\|_{K_{a}}=\|x-a\|_{K-a}=\inf \{\lambda: x-a \in \lambda(K-a)\} .
$$

We denote $X_{a}=\left(X,\|\cdot\|_{K_{a}}\right)$; this could be further identified with $V_{a}=$ $\left(V, p_{K, a}\right)$. The definition of the Banach-Mazur distance generalizes immediately to spaces with a possibly non-symmetric gauge function and so we will write $d_{K_{a}}=d_{X_{a}}=d\left(l_{2}^{n}, X_{a}\right)$. However, in the affine context it is more 
natural to consider affine rather than linear maps; if $K, K^{\prime}$ are subsets of an affine space this leads to the definition of the "affine Banach-Mazur distance" $\hat{d}\left(K, K^{\prime}\right):=\inf _{a, b} d\left(K_{a}, K_{b}^{\prime}\right)$ and to $\hat{d}_{K}:=\inf _{a} d\left(l_{2}^{n}, X_{a}\right)=\inf _{a} d_{X_{a}}$, the latter necessarily $\leq n$ (see John (1948)).

The affine space in question will be usually endowed with a Euclidean structure, in particular we may talk about polars of $K_{a}$ 's (or of $K$ with respect to its interior point $a$ ) and the functionals $\ell_{p}\left(K_{a}\right)$. We denote by $G L(X)$ the group of automorphisms of the vector space $X$ (for fixed $a \in K$, one may also think of $G L(X)$ as of affine maps preserving $a$ ). We will prove

Theorem 4.1 There exists an absolute constant $C$ such that for any convex body $K$ in an $n$-dimensional (affine) Euclidean space

$$
\operatorname{Ell}(K) \equiv \inf _{a \in K, w \in G L(X)}\left\{\ell\left(w K_{a}\right) \cdot \ell\left(\left(w K_{a}\right)^{\circ}\right)\right\} \leq C n \sqrt{\hat{d}_{K}}
$$

As in the symmetric case, we are going to use the Gaussian projection. When considered as operating on $L_{2}\left(X_{a}\right)$, we shall denote it by $G_{a}$. However, we point out that the underlying vector space being the same for all $a$ 's, $G_{a}$ as a linear map does not depend on $a$. Moreover, $G$ (or $G_{a}$ ) annihilates constant functions and so we may as well talk about $G_{a} f$ if $f$ is affine space valued.

If we now use the usual operator norm and define the functional

$$
\psi(K)=\psi_{G}(K):=\inf _{a \in K} \sup _{f \neq 0} \frac{\left\|G_{a} f\right\|_{L_{2}\left(X_{a}\right)}}{\|f\|_{L_{2}\left(X_{a}\right)}},
$$

then easy examples show that

$$
\psi_{G}(K) \geq c \hat{d}(K, K \cap-K)
$$

and $\hat{d}(K, K \cap-K)$ can be as large as $n$ (as can be checked directly for the simplex, or see Grünbaum (1963) for this and a general discussion of related matters). To obtain a satisfactory result we are going to define new functionals, suggested by E. Gluskin, which will replace the standard norm of an operator. Let

$$
\varphi(K)=\varphi_{G}(K):=\sup _{f \neq 0} \inf _{a \in K} \frac{\left\|G_{a} f\right\|_{L_{2}\left(X_{a}\right)}}{\|f\|_{L_{\infty}\left(X_{a}\right)}},
$$




$$
\varphi_{2}(K)=\varphi_{2, G}(K):=\sup _{f \neq 0} \inf _{a \in K} \frac{\left\|G_{a} f\right\|_{L_{2}\left(X_{a}\right)}}{\|f\|_{L_{2}\left(X_{a}\right)}} .
$$

Note that considering $G_{a}$ as an operator $G_{a}: L_{\infty} \rightarrow L_{2}$ rather than $G_{a}$ : $L_{2} \rightarrow L_{2}$ is quite natural as the inequality

$$
\|F\|_{L_{\infty}\left(X_{a}\right)} \leq 1
$$

has a simple geometric meaning, independent of the choice of the origin $a \in K$ : it just means that $F(t) \in K$ a.e. in $\Omega$. For symmetric bodies all these three functionals are still the usual norms; moreover, $\psi(\cdot)=\varphi_{2}(\cdot)$ while $\varphi(K)$ and $\varphi_{2}(K)$ are equivalent up to a universal multiplicative constant (the latter follows by considering $G$ as acting from $L_{2}$ to $L_{1}$-i.e., as the adjoint to $G: L_{\infty} \rightarrow L_{2}$ - and using the fact that the $L_{2}$ and $L_{1}$ norms are equivalent on the range of $G$ by Lemma 3.3, the Khinchine-Kahane inequality). For non-symmetric bodies the difference between $\varphi(K)$ and $\psi(K)$ becomes substantial. For example, in the case of the $n$-dimensional simplex $S$ one can prove that (see Proposition 5.5 in the last section)

$$
\varphi(S) \leq C(1+\log n)
$$

which implies the same sharp estimate for $\operatorname{Ell}(S) \leq C n(1+\log n)$ as that given by Theorem 2.4 or Proposition 3.1, while

$$
\psi(S) \geq c n .
$$

Still, the new functional satisfies the Figiel-Tomczak-Jaegermann type estimate. We have

Proposition 4.2 Under the assumptions of Theorem 4.1 there is an absolute constant $C$ such that

$$
\operatorname{Ell}(K) \equiv \inf _{a \in K, w \in G L(X)}\left\{\ell\left(w K_{a}\right) \cdot \ell\left(\left(w K_{a}\right)^{\circ}\right)\right\} \leq C n \varphi(K)
$$

For reader's convenience we sketch the proof, which mimics that of the symmetric case (Figiel and Tomczak-Jaegermann (1979), see also Bourgain and Milman (1987)), at the end of this section. Unfortunately, we were not able to obtain the logarithmic estimate for $\varphi(K)$ in the general case. The following result gives some non-trivial estimate. 
Proposition 4.3 For any convex body $K$ we have

$$
\varphi(K) \leq \varphi_{2}(K) \leq 9 \sqrt{\hat{d}_{K}}
$$

Clearly the last two Propositions imply Theorem 4.1. The proof of Proposition 4.3 consists of two parts. First, we estimate the norm of the Gaussian projection of a mean zero function. We then handle arbitrary functions by properly choosing the center.

Lemma 4.4 Let $q$ be a (possibly non-symmetric) gauge function on $\mathbb{R}^{n}$ and let $X=\left(\mathbb{R}^{n}, q\right)$. Let $f$ be function in $L_{2}(X)$, such that $\int_{\Omega} f(t) d P=0$. Then

$$
\|G f\|_{L_{2}(X)} \leq\left(1+2 \sqrt{d_{X}}\right)\|f\|_{L_{2}(X)} .
$$

We will mimic the proof of the analogous statement in Pisier (1989), Theorem 2.5. However, in the non-symmetric case we can not use the Bernstein's inequality, so we replace Lemma 2.6 of Pisier (1989) by the following

Lemma 4.5 Let $\left\{x_{k}: k \geq 1\right\}$ be a finitely supported sequence in a linear space equipped with a convex positively homogeneous non-negative functional q. Assume that

$$
\max _{0<\varepsilon \leq 1 / 2} q\left(\sum_{1}^{\infty} \varepsilon^{k} x_{k}\right) \leq 1
$$

and set $\Delta=\max _{k \geq 2} q\left(-x_{k}\right)$. Then

$$
q\left(x_{1}\right) \leq 1+2 \sqrt{\max \{1, \Delta\}}
$$

Proof. By homogeneity and convexity of the functional $q$ we have

$$
q\left(x_{1}\right) \leq \varepsilon^{-1}\left(q\left(\sum_{1}^{\infty} \varepsilon^{k} x_{k}\right)+q\left(\sum_{2}^{\infty}-\varepsilon^{k} x_{k}\right)\right) \leq \varepsilon^{-1}\left(1+\frac{\varepsilon^{2} \Delta}{1-\varepsilon}\right) .
$$

Setting $\varepsilon=\min \left\{1 / 2,(1+\sqrt{\Delta})^{-1}\right\}$ completes the proof.

Proof of Lemma 4.4. As in Pisier (1989), for $\varepsilon \in[-1,1]$ consider the operator

$$
T_{\varepsilon}:=\sum_{k \geq 0} \varepsilon^{k} Q_{k}: L_{2} \rightarrow L_{2}
$$


where, for $k=0,1,2, \ldots, Q_{k}$ is the orthogonal projection onto the linear span of Hermite polynomials of degree exactly $k$ that was defined earlier. Let $S=\operatorname{span}\left\{H_{\alpha}: \alpha \in A\right\} \subset L^{2}=L^{2}(\Omega, P)$ be the linear span of all Hermite polynomials. It was shown in Pisier (1989), Lemma 2.1 that, for $f \in S$,

$$
T_{\varepsilon} f(x)=\int_{\Omega} f\left(\varepsilon u+\sqrt{1-\varepsilon^{2}} v\right) d P(y) .
$$

Take $f$ in $S \otimes X$ with $\|f\|_{L_{2}(X)} \leq 1$ and let $f_{\varepsilon}(u, v):=f\left(\varepsilon u+\sqrt{1-\varepsilon^{2}} v\right)$. Now, in the finite-dimensional case, $\left(L_{2}(X)\right)^{*}=L_{2}\left(X^{*}\right)$, where $X^{*}=$ $\left(\mathbb{R}^{n}, q_{*}\right), q_{*}=\|\cdot\|_{K^{\circ}}$ being the gauge dual to $q=\|\cdot\|_{K}$. Consequently, there exists a function $g \in L_{2}\left(X^{*}\right)$ such that $\|g\|_{L_{2}\left(X^{*}\right)}=1$ and

$$
\begin{aligned}
& \left\|\left(T_{\varepsilon} \otimes I_{X}\right) f\right\|_{L_{2}(X)}=\int_{\Omega}\left\langle\left(T_{\varepsilon} \otimes I_{X}\right) f, g\right\rangle d P \\
= & \int_{\Omega} \int_{\Omega}\left\langle f_{\varepsilon}(u, v), g(x)\right\rangle d P(y) d P(x) \\
\leq & \left(\int_{\Omega} \int_{\Omega} q^{2}\left(f_{\varepsilon}(u, v)\right) d P(y) d P(x)\right)^{1 / 2}\left(\int_{\Omega} \int_{\Omega} q_{*}^{2}(g(x)) d P(y) d P(x)\right)^{1 / 2} \\
= & \|f\|_{L_{2}(X)}\|g\|_{L_{2}\left(X^{*}\right)} \leq 1 .
\end{aligned}
$$

The last equality is implied by the fact that, for any $\varepsilon \in[-1,1]$, the function $f_{\varepsilon}(u, v) \equiv f\left(\varepsilon u+\sqrt{1-\varepsilon^{2}} v\right)$ on $(\Omega \times \Omega, P \otimes P)$ has the same distribution as $f$ on $(\Omega, P)$ (which in turn follows from $\sum \alpha_{i} g_{i}$ having the same distribution as $\left.\sqrt{\sum \alpha_{i}^{2}} g_{1}\right)$. Clearly

$$
\left\|\left(Q_{k} \otimes I_{X}\right) f\right\|_{L_{2}(X)} \leq d_{X}
$$

as in the symmetric case (see Pisier (1989), Lemma 2.2). Therefore, applying Lemma 4.5 with $\Delta=d_{X}, B=L_{2}(X)$ and $x_{k}=\left(Q_{k} \otimes I_{X}\right) f$, we obtain

$$
\|G f\|_{L_{2}(X)} \equiv\left\|\left(Q_{1} \otimes I_{X}\right) f\right\|_{L_{2}(X)} \leq 1+2 \sqrt{d_{X}} .
$$

As $S \otimes X$ is dense in $L_{2}(X)$, Lemma 4.4 follows.

Proof of Proposition 4.3: Let $K \subset \mathbb{R}^{n}$ (thought of as both an affine and a vector space) be a convex body such that $d_{K_{0}}=\hat{d}_{K}$. Fix $f \in L_{2}\left(\mathbb{R}^{n}\right)$ and set $x_{\emptyset}=Q_{0} f=\int_{\Omega} f d P$. If $x_{\emptyset} \neq 0$, we shall, for this particular $f$, choose the origin $a$ as

$$
a=\frac{c_{1}-c_{2}}{2} x_{\emptyset}, \quad \text { where } c_{1}^{-1}=\left\|x_{\emptyset}\right\|_{K_{0}}, c_{2}^{-1}=\left\|-x_{\emptyset}\right\|_{K_{0}} .
$$



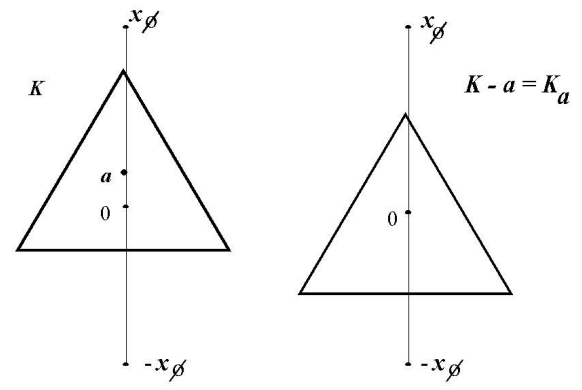

Figure 1: Choosing the origin.

In other words, $a$ is chosen to be the midpoint of the segment

$$
K_{0} \cap\left\{\lambda x_{\emptyset}: \lambda \in \mathbb{R}\right\}
$$

(see Figure 1). Then $\left\|x_{\emptyset}\right\|_{K_{a}}=\left\|-x_{\emptyset}\right\|_{K_{a}}$ and $a \in \frac{1}{2} K_{0}$; the latter implies that $d_{K_{a}} \leq 3 d_{K_{0}}=3 \hat{d}_{K}$. (Indeed, if $\mathcal{E}$ is an ellipsoid such that $\mathcal{E} \subset K_{0} \subset d_{K_{0}} \mathcal{E}$, then $\frac{1}{2} \mathcal{E} \subset \frac{1}{2} K_{0} \subset K_{a}=K_{0}-a \subset \frac{3}{2} d_{K_{0}} \mathcal{E}$.) These assertions hold also, trivially, if $x_{\emptyset}=0$ and we set $a=0$. Thus, by Lemma 4.4,

$$
\begin{aligned}
\left\|G_{a} f\right\|_{L_{2}\left(X_{a}\right)} & \leq\left(1+2 \sqrt{d_{X_{a}}}\right)\left\|f-x_{\emptyset}\right\|_{L_{2}\left(X_{a}\right)} \\
& \leq\left(1+2 \sqrt{d_{X_{a}}}\right)\left(\|f\|_{L_{2}\left(X_{a}\right)}+\left\|-x_{\emptyset}\right\|_{L_{2}\left(X_{a}\right)}\right) \\
& \leq\left(1+2 \sqrt{3 \hat{d}_{K}}\right)\left(\|f\|_{L_{2}\left(X_{a}\right)}+\left\|x_{\emptyset}\right\|_{K_{a}}\right) \\
& \leq\left(2+4 \sqrt{3 \hat{d}_{K}}\right)\|f\|_{L_{2}\left(X_{a}\right)} .
\end{aligned}
$$

The last inequality uses the fact that $\left\|x_{\emptyset}\right\|_{K_{a}} \leq\|f\|_{L_{2}\left(X_{a}\right)}$, which follows just from Jensen's inequality. This completes the proof of the Proposition.

For the Proof of Proposition 4.2 we need 
Lemma 4.6 Lewis (1979) Let $\alpha$ be a gauge function on $n \times n$ matrices which vanishes only at 0 and let $\alpha_{*}$ be the dual gauge function (with respect to the trace duality i.e., given by $\langle u, v\rangle=\operatorname{tr}(u v))$. Then there exists $u \in G L(n)$ such that $\alpha(u)=1$ and $\alpha_{*}\left(u^{-1}\right)=n$.

The above result is stated in Lewis (1979) for (ideal) norms only, but the argument does not use symmetry (nor, a priori, any ideal property).

Proof of Proposition 4.2. As before, we may assume that $d_{K_{0}}=d_{K}$, in particular 0 is an interior point of $K$. We will show that, for some $w \in G L(n)$ and $a \in K$,

$$
\ell_{1}\left(\left(w K_{a}\right)^{\circ}\right) \leq n
$$

while

$$
\ell\left(w K_{a}\right) \leq \varphi(K) \equiv \sup _{f \neq 0} \inf _{a \in K} \frac{\left\|G_{a} f\right\|_{L_{2}\left(X_{a}\right)}}{\|f\|_{L_{\infty}\left(X_{a}\right)}} .
$$

By Lemma 3.3, these two inequalities imply Proposition 4.2.

As before, let $g_{1}, g_{2}, \ldots$ be independent identically distributed standard Gaussian random variables and $G=G_{0}$ the (usual linear) Gaussian projection. As only $g_{j}, j=1, \ldots, n$, will be relevant, we may replace $(\Omega, P)$ by $\left(\mathbb{R}^{n}, d \gamma_{n}\right)$ (applying the conditional expectation when necessary). Identifying $X=X_{0}$ with $\mathbb{R}^{n}$, we define a gauge function $\alpha$ on the space of $n \times n$ matrices by

$$
\alpha(u)=\inf \left\{\|f\|_{L_{\infty}(X)}: G f=\sum_{i=1}^{n} u e_{i} g_{i}\right\} .
$$

By Lemma 4.6, there exists an isomorphism $u: \mathbb{R}^{n} \rightarrow X$ such that $\alpha(u)=1$ and $\alpha_{*}\left(u^{-1}\right)=n$. In particular, there exists a function $f \in L_{\infty}(X)$ such that

$$
\|f\|_{L_{\infty}(X)}=1 \text { and } G f=\sum_{i=1}^{n} u e_{i} g_{i}
$$

(Here and throughout the rest of this argument we take for granted that the infima/suprema in question are achieved. This is true because of $w^{*}$ compactness, or we could have worked with objects "almost achieving" them.) Hence, by the definition of $\varphi(\cdot)$, there exists $a \in K$ such that

$$
\varphi(K) \geq\left\|G_{a} f\right\|_{L_{2}\left(X_{a}\right)}=\left(\int\left\|\sum_{i=1}^{n} u e_{i} g_{i}\right\|_{K_{a}}^{2} d \gamma_{n}\right)^{1 / 2}=\ell\left(u^{-1} K_{a}\right)
$$


which yields the inequality (\#\#) with $w=u^{-1}$. On the other hand,

$$
\ell_{1}\left(\left(u^{-1} K_{a}\right)^{\circ}\right)=\ell_{1}\left(\left(u^{-1} K\right)^{\circ}\right)=\ell_{1}\left(u^{*} K^{\circ}\right)=\int\left\|\sum_{i=1}^{n}\left(\left(u^{*}\right)^{-1} e_{i}\right) g_{i}\right\|_{K^{\circ}} d \gamma_{n}
$$

as, by the second assertion of Lemma $2.2, \ell_{1}\left(\left(u^{-1} K_{a}\right)^{\circ}\right)=\ell_{1}\left(\left(u^{-1} K-u^{-1} a\right)^{\circ}\right)$ does not depend on the choice of $a \in K$ and since $\left(u^{-1} K\right)^{\circ}=u^{*} K^{\circ}$. Now choose $h \in L_{\infty}(X)$ of norm one such that the last integral equals

$$
\int\left\langle\sum_{i=1}^{n}\left(\left(u^{*}\right)^{-1} e_{i}\right) g_{i}, h\right\rangle d \gamma_{n}=\sum_{i=1}^{n}\left\langle\left(u^{*}\right)^{-1} e_{i}, z_{i}\right\rangle=\sum_{i=1}^{n}\left\langle e_{i}, u^{-1} z_{i}\right\rangle
$$

where $z_{i}=\int g_{i} h d \gamma_{n}$.

Define $v: l_{2}^{n} \rightarrow X$ by $v e_{i}=z_{i}$. By definition, $\alpha(v) \leq 1$, and so

$$
\sum_{i=1}^{n}\left\langle e_{i}, u^{-1} z_{i}\right\rangle=\operatorname{tr}\left(u^{-1} v\right) \leq \alpha_{*}\left(u^{-1}\right) \alpha(v) \leq n
$$

which is (\#) with $w=u^{-1}$. This completes the proof.

\section{The logarithmic factors - do we need them?}

As described in the introduction, the invariant Flt $(\cdot)$ in dimension $n$ verifies $c n \leq \operatorname{Flt}(\cdot) \leq C n(1+\log n)$ for symmetric bodies (Banaszczyk (1996)), and it is conceivable that similar estimates hold in the non-symmetric case, even though our argument gives, in general, a significantly weaker upper estimate. There is no substantial evidence as to whether the logarithmic factor is necessary. On the other hand, one can not hope to remove that factor by estimating more precisely the invariant $\operatorname{Ell}(\cdot)$. We have

Proposition 5.1 Let $S$ and $Q$ denote respectively an $n$-dimensional simplex and cube. Then

$$
\begin{gathered}
\operatorname{Ell}(Q) \geq c n(1+\log n)^{1 / 2}, \\
\operatorname{Ell}(S) \geq c^{\prime} n(1+\log n),
\end{gathered}
$$

where $c$ and $c^{\prime}$ are universal positive constants. 
Thus the lower estimates are of the same order as the upper estimates implied by the (asymptotically) known respective $K$-convexity constants (see Banaszczyk (1996) for the cube and Proposition 5.5 below, combined with Proposition 4.2, for the simplex) or, even simpler, by direct calculation. This equivalence is not really surprising, but we provide a proof as we could not find a reference in the literature. The argument clearly works in a much more general setting (e.g., for spaces or bodies "with enough symmetries", see Tomczak-Jaegermann (1989), §16), but - in particular - we haven't attempted to verify whether examples due to Bourgain (1984) of $n$-dimensional spaces whose $K$-convexity constants are $O(1+\log n)$ yield Ell(.) of order $n(1+\log n)$. The proof is based on the following two elementary facts.

Lemma 5.2 Let $K_{1}, K_{2} \in \mathcal{K}^{n}, w \in G L(n)$ and $p \in[1, \infty)$. Then there exist linear subspaces $E_{1}, E_{2} \subset \mathbb{R}^{n}$ with $\operatorname{dim} E_{i}=m_{i} \geq n / 2, i=1,2$, verifying

$$
\ell_{p}\left(E_{1} \cap K_{1}\right) \cdot \ell_{p}\left(E_{2} \cap K_{2}^{\circ}\right) \leq \ell_{p}\left(w K_{1}\right) \cdot \ell_{p}\left(\left(w K_{2}\right)^{\circ}\right) .
$$

We point out that the quantities $\ell_{p}(\cdot)$ in the assertion refer to averages with respect to the standard Gaussian measures on the respective spaces $E_{i}$. We emphasize that the argument does not require symmetry of the gauge function.

Proof. For clarity, we will present the proof for $p=1$, the argument for other $p$ 's is identical. We have $\|x\|_{w K_{1}}=\left\|w^{-1} x\right\|_{K_{1}}$ and, considering that $\left(w K_{2}\right)^{\circ}=\left(w^{*}\right)^{-1} K_{2}^{\circ},\|x\|_{\left(w K_{2}\right)^{\circ}}=\left\|w^{*} x\right\|_{K_{2}^{\circ}}$. Let $w^{-1}=U \Delta$ be the polar decomposition of $w^{-1}$, i.e., $U \in O(n), \Delta$ positive. Replacing, if necessary, $K_{i}$ by $U^{*} K_{i}$, we may assume that $U$ is the identity, hence $w^{-1}=\Delta$ and $w^{*}=\Delta^{-1}$. Let $s_{1} \geq s_{2} \geq \ldots \geq s_{n}>0$ be the eigenvalues of $\Delta$ and let $\left(u_{k}\right)$ the corresponding orthonormal basis of $\mathbb{R}^{n}$ diagonalizing $\Delta$. Set $m=m_{1}=$ $\lceil n / 2\rceil$ and let $E_{1}=\operatorname{span}\left\{u_{k}: 1 \leq k \leq m\right\}$ and $E_{2}=\operatorname{span}\left\{u_{k}: m \leq k \leq n\right\}$ respectively. Clearly $\operatorname{dim} E_{1}, \operatorname{dim} E_{2} \geq n / 2$ while

$$
s_{m} \int_{E_{1}}\|x\|_{K_{1}} d \gamma_{m}(x) \leq \int_{E_{1}}\|\Delta x\|_{K_{1}} d \gamma_{m}(x) \leq \int_{\mathbb{R}^{n}}\|\Delta x\|_{K_{1}} d \gamma_{n}(x)=\ell_{1}\left(K_{1}\right) .
$$

Both inequalities above follow from the "ideal property" of the $\ell$-norm, i.e., $\int\|v x\|_{K} d \gamma(x) \leq \int\|x\|_{K} d \gamma(x)$ if $v$ is a contraction on the Euclidean space (see e.g., Pisier (1989), (3.12); the argument does not use symmetry of the 
gauge function), the first one applied to $v=s_{m} \Delta_{\mid E_{1}}^{-1}$, and the second to the orthogonal projection onto $E_{1}$. Similarly,

$$
s_{m}^{-1} \int_{E_{2}}\|x\|_{K_{2}^{\circ}} d \gamma_{m_{2}}(x) \leq \int_{\mathbb{R}^{n}}\left\|\Delta^{-1} x\right\|_{K_{2}^{\circ}} d \gamma_{n}(x)=\ell_{1}\left(K_{2}^{\circ}\right),
$$

whence the Lemma follows.

Before stating the next lemma we recall the definition of the $\ell$-ellipsoid, already implicitly referred to several times in the paper. Given $K \in \mathcal{K}^{n}$, let $u \in G L(n)$ be given by Lemma 4.6 applied to $\alpha(v)=\ell(v) \equiv \ell\left(v^{-1} K\right)=$ $\left(\int_{\mathbb{R}^{n}}\|v x\|_{K}^{2} d \gamma_{n}(x)\right)^{1 / 2}$, we then set $\mathcal{E}=u B_{2}^{n}$, where $B_{2}^{n}$ is the Euclidean unit ball. We need to know that (i) $\mathcal{E}$ is unique and (ii) any $u$ giving $\mathcal{E}$ maximizes $|\operatorname{det} u|$ over $\{u: \ell(u) \leq 1\}$. We have

Lemma 5.3 Let $K \in \mathcal{K}^{n}$ be such that its $\ell$-ellipsoid $K$ is the unit Euclidean ball. Let $E \subset \mathbb{R}^{n}$ be a linear subspace, $\operatorname{dim} E=m$. Denote $\theta=m / n$. Then

$$
\ell(E \cap K) \geq \theta(1-\theta)^{\frac{1-\theta}{\theta}} .
$$

In particular if $\operatorname{dim} E \geq n / 2$, then $\ell(E \cap K) \geq 1 / 4$.

Proof. (Sketch) Let $P$ be the orthogonal projection onto $E$, then $\ell(P) \leq$ $\ell(I)=1$ by the ideal property of the $\ell$-norm (see the proof of the preceding lemma), similarly $\ell(I-P) \leq 1$. Consider now, for $s, t>0, u=s P+t(I-P)$. One shows that if the condition in the assertion of the Lemma was not satisfied, one would be able to choose $s, t$ so that $\ell(u) \leq s \ell(P)+t \leq$ $s \ell(E \cap K)+t=1$ while $|\operatorname{det} u|=s^{m} t^{n-m}>1=|\operatorname{det} I|$, contradicting the optimal property of the identity $I$. More precisely, the same argument gives $\ell(P)^{\theta} \ell(I-P)^{1-\theta} \geq \theta^{\theta}(1-\theta)^{1-\theta}$.

Proof of Proposition 5.1. One checks directly that for the cube $Q$ in the "standard" position, i.e., $Q=[-1,1]^{n} \subset \mathbb{R}^{n}, \ell(Q)$ is precisely of order $(1+\log n)^{1 / 2}$ while $n>\ell\left(Q^{\circ}\right) \geq \ell_{1}\left(Q^{\circ}\right)=(2 / \pi)^{1 / 2} n$. Now the $\ell$-ellipsoid of $Q$ (and its polar) is a multiple of the Euclidean ball; this follows from the fact that these are the only ellipsoids which are invariant under the group of symmetries of $Q$ (resp. its polar) and from the uniqueness of the $\ell$-ellipsoid. Hence, by Lemma 5.3, if $\operatorname{dim} E \geq n / 2$, then $\ell(Q \cap E) \geq \ell(Q) / 4$ (resp. $\left.\ell\left(Q^{\circ} \cap E\right)\right) \geq \ell\left(Q^{\circ}\right) / 4$ ). Therefore, by Lemma 5.2 (applied with 
$\left.K_{1}=K_{2}=Q\right)$, for any $w \in G L(n), \ell(w Q) \cdot \ell\left((w Q)^{\circ}\right) \geq c n(1+\log n)^{1 / 2}$, as required.

Let $S$ be a regular simplex in the "standard" position, i.e., with centroid at the origin and, say, circumscribed to the Euclidean unit ball. It is rather easy to check that $\ell(S)$ is precisely of order $(1+\log n)^{1 / 2}$ and $\ell\left(S^{\circ}\right)=n \ell(S)$ (but we will not use this information explicitly.) The same argument as applied above to $Q$ works for $S$ provided we restrict ourselves to linear maps. However, in the general affine case we "loose control" of the $\ell$-ellipsoid of $S-a$ and so we have to be more careful. Let $w \in G L(n)$ and let $a$ be an interior point of $S$. Denote $\lambda=\ell(w(S-a)) \cdot \ell\left((w(S-a))^{\circ}\right)$. Now, by Lemma $2.2, \ell_{1}\left((K-a)^{\circ}\right)$ does not depend on $a$ and so $\ell\left((w(S-a))^{\circ}\right)=$ $\ell\left((w S-w a)^{\circ}\right) \geq \ell_{1}\left((w S-w a)^{\circ}\right)=\ell_{1}\left((w S)^{\circ}\right) \geq c_{0} \ell\left((w S)^{\circ}\right)$, where the last inequality uses Lemma 3.3. Hence

$$
\ell(w(S-a)) \cdot \ell\left((w S)^{\circ}\right) \leq c_{0}^{-1} \lambda .
$$

We now are in a position to apply Lemma 5.2 with $K_{1}=S-a$ and $K_{2}=S$; the Proposition will follow if we show that, for linear subspaces $E_{1}, E_{2} \subset \mathbb{R}^{n}$ with $\operatorname{dim} E_{i} \geq n / 2, i=1,2$,

$$
\ell\left(E_{1} \cap(S-a)\right) \cdot \ell\left(E_{2} \cap S^{\circ}\right) \geq c_{1} n(1+\log n) .
$$

For our representation of $S$ (circumscribed to the Euclidean unit ball), let $u_{i}, i=1, \ldots, n+1$, be the contact points. Then $S=\left\{x \in \mathbb{R}^{n}:\left\langle x, u_{i}\right\rangle \leq\right.$ 1 for $i=1, \ldots, n+1\}$ and $v_{i}=-n u_{i}$ are the vertices of $S$. In particular, it follows that $S^{\circ}=-S / n$, hence $\|x\|_{S^{\circ}}=n\|-x\|_{S}$. Accordingly $\ell\left(E_{2} \cap S^{\circ}\right)=$ $n \ell\left(E_{2} \cap S\right)$ and so, to show the last inequality, it clearly suffices to establish that if $a$ is an interior point of $S$ and $\operatorname{dim} E \geq n / 2$, then

$$
\ell(E \cap(S-a)) \geq c_{2}(1+\log n)^{1 / 2} .
$$

To this end observe first that the vectors $x \in \mathbb{R}^{n}$ are uniquely represented as $x=\sum_{i=1}^{n+1} t_{i} v_{i}$ with $\sum_{i=1}^{n+1} t_{i}=0$, moreover, one has $t_{i}=-\frac{\left\langle x, u_{i}\right\rangle}{n+1}$ for $i=1, \ldots, n+1$. For such an $x$ and for the choice of the point $a=\sum_{i=1}^{n+1} a_{i} v_{i} \in$ $S$ (with $\sum_{i=1}^{n+1} a_{i}=1, a_{i} \geq 0$ ) as the origin, one has

$$
\|x\|_{S-a}=\left\|\sum_{i=1}^{n+1} t_{i} v_{i}\right\|_{S-a}=\max _{i \leq n+1}\left(-\frac{t_{i}}{a_{i}}\right)=\max _{i \leq n+1}\left\langle x, \frac{u_{i}}{(n+1) a_{i}}\right\rangle .
$$

(We employ here the notation $\|x\|_{S-a}$ rather than $p_{S_{a}}(x)$ as $x$ is a vector rather than a point in an affine space.) Now the contact points $\left(u_{i}\right)$ yield 
a "decomposition of identity" as in Lemma 3.4 (this has been already used above), one has

$$
\forall x \in \mathbb{R}^{n}, x=\frac{n}{n+1} \sum_{i=1}^{n+1}\left\langle x, u_{i}\right\rangle u_{i} .
$$

A well-known argument (see e.g., Pełczyński (1980), Prop. 16.1) shows then that, for any rank $k$ orthogonal projection $P$ in $\mathbb{R}^{n}$, one has $\max _{i \leq n+1}\left|P u_{i}\right| \geq$ $(k / n)^{1 / 2}$. Thus, starting with an arbitrary subspace $E \subset \mathbb{R}^{n}$ with $\operatorname{dim} E=$ $m \geq n / 2$, one can construct inductively a sequence $u_{i_{1}}, u_{i_{2}}, \ldots, u_{i_{s}}, s \geq n / 4$, such that, denoting by $P_{E}$ the orthogonal projection onto $E$ and $w_{j}=P_{E} u_{i_{j}}$, one has, for $j=1, \ldots, s,\left|w_{j}\right| \geq 1 / 2$ and, moreover,

$$
\operatorname{dist}\left(w_{j}, \operatorname{span}\left\{w_{r}: 1 \leq r<j\right\}\right) \geq 1 / 2 .
$$

Recall that, for a vector $x \in E$,

$$
\|x\|_{S-a}=\max _{i \leq n+1}\left\langle x, \frac{u_{i}}{(n+1) a_{i}}\right\rangle=\max _{i \leq n+1}\left\langle x, \frac{P_{E} u_{i}}{(n+1) a_{i}}\right\rangle \geq \max _{j \leq s}\left\langle x, \frac{w_{j}}{(n+1) a_{i_{j}}}\right\rangle .
$$

As $\sum_{i=1}^{n+1} a_{i}=1$, it follows that among the numbers $a_{i}$, strictly less than $(n+1) / 8$, hence at most $n / 8$, are $>8 /(n+1)$, and so the set $J=\left\{j \leq s: a_{i_{j}}<8 /(n+1)\right\}$, is of cardinality at least $n / 8$ (remember $s \geq n / 4)$. Denoting $y_{j}=w_{j} /\left((n+1) a_{i_{j}}\right)$ we see from the preceding inequality that, for $j \in J,\left|y_{j}\right|>1 / 16$ and, moreover, $\left|y_{j}-y_{j^{\prime}}\right| \geq 1 / 16$ for $j, j^{\prime} \in J, j \neq j^{\prime}$. To summarize, we have shown that there exist $y_{j} \in E$ for $j \in J$ with

(1) $\|x\|_{S-a} \geq \max _{j \in J}\left\langle x, y_{j}\right\rangle$

(2) $\left|y_{j}-y_{j^{\prime}}\right| \geq 1 / 16$ for $j, j^{\prime} \in J, j \neq j^{\prime}$

(3) the cardinality of $J$ is at least $n / 8$.

This type of situation is handled by one of the companion inequalities to Lemma 3.5, the "Sudakov minoration" (see e.g., Pisier (1989), Theorem 5.6).

Lemma 5.4 Let $\alpha>0$ and let $Z_{1}, \ldots, Z_{M}$ be a sequence of jointly Gaussian random variables on a probability space $(\Omega, P)$ such that

$$
\left\|Z_{j}-Z_{j^{\prime}}\right\|_{L_{2}(\Omega)} \geq \alpha \text { for } j, j^{\prime} \in\{1, \ldots, M\}, j \neq j^{\prime} .
$$

Then

$$
\int_{\Omega} \max _{1 \leq j \leq M} Z_{j} d P \geq c_{1} \alpha(1+\log M)^{1 / 2}
$$

where $c_{1}$ is a universal constant. 
As the map $E \ni y \rightarrow\langle\cdot, y\rangle \in L_{2}\left(E, \gamma_{m}\right)$ is an isometry, Lemma 5.4 combined with (1)-(3) implies that

$$
\ell((S-a) \cap E) \geq \ell_{1}((S-a) \cap E)=\int_{E}\|x\|_{S-a} d \gamma_{m}(x) \geq \frac{c_{1}}{16}\left(1+\log \frac{n}{8}\right)^{1 / 2},
$$

whence $(\dagger)$ easily follows.

As indicated earlier, the asymptotically precise estimate $\operatorname{Ell}(S) \leq$ $C n(1+\log n)$, where $S$ is an $n$-dimensional simplex, is not difficult to obtain by direct calculation (applied to the regular simplex). It also follows from the methods of section 3 (Proposition 3.1). Finally, it may be alternatively derived from Proposition 4.2 and the following result, which we include primarily as it suggests that the non-symmetric analogues of the $K$-convexity constants introduced in section 4 are, in some sense, correct.

Proposition 5.5 Let $S$ be an n-dimensional simplex. Then

$$
c(1+\log n) \leq \varphi(S) \leq C(1+\log n),
$$

where $c$ and $C$ are universal positive constants.

Proof. The first inequality follows directly from Proposition 5.1 and Proposition 4.2. As in the proof of Proposition 5.1, let $S$ be a regular simplex circumscribed to the Euclidean unit ball; we will keep the notation of that proof in the sequel. Clearly, it is enough to prove the claim for this particular $n$-dimensional simplex.

By the definition of the invariant $\varphi(\cdot)$ and the comments following it, given $F: \Omega \rightarrow S$, we need to estimate (by $C(1+\log n)$ ) the quantity $\inf _{a \in S}\left\|G_{a} F\right\|_{L_{2}\left(X_{a}\right)} . \quad F$ being $S$-valued, there exist functions $f_{1}, \ldots, f_{n+1}$ : $\Omega \rightarrow \mathbb{R}^{+}$such that $\sum_{i=1}^{n+1} f_{i} \equiv 1$ and $F=\sum_{i=1}^{n+1} f_{i} v_{i}$. The Gaussian projection of $F$ is now just $\sum_{i=1}^{n+1} G f_{i} v_{i}$, where $G$ is the usual Gaussian projection (defined for scalar valued functions); we point out that $\sum_{i=1}^{n+1} G f_{i} \equiv 0$ and so, as explained in the proof of Proposition 5.1, $\sum_{i=1}^{n+1} G f_{i} v_{i}$ represents a vector function, whose $L_{2}$-norm with respect to the gauge $\|\cdot\|_{S-a}$ (i.e. $\left\|G_{a} F\right\|_{L_{2}\left(X_{a}\right)}$ in the language of section 4 , the quantity we need to estimate) is

$$
A=\left(\int_{\Omega}\left|\max _{i}\left(-\frac{G f_{i}}{a_{i}}\right)\right|^{2} d t\right)^{1 / 2} .
$$


By Lemma 3.3 with $p=2$ and by Lemma 3.5 applied with $Y_{i}=-G f_{i} / a_{i}$ (which are Gaussian random variables), we have

$$
A \leq \sqrt{2} c^{-1} \int_{\Omega} \max _{i}\left|\frac{G f_{i}}{a_{i}}\right| d P \leq C^{\prime}(1+\log n)^{1 / 2} \max _{i} \frac{\left\|G f_{i}\right\|_{2}}{a_{i}} .
$$

Now if $a=\left(a_{1}, \ldots, a_{n+1}\right) \in S$ is given by $a_{i}=\left\|G f_{i}\right\|_{2} /\left(\sum_{i}\left\|G f_{i}\right\|_{2}\right)$, we get

$$
A \leq C^{\prime}(1+\log n)^{1 / 2} \sum_{i}\left\|G f_{i}\right\|_{2}=C^{\prime}(\pi / 2)^{1 / 2}(1+\log n)^{1 / 2} \sum_{i}\left\|G f_{i}\right\|_{1} .
$$

On the other hand

$\sum_{i}\left\|G f_{i}\right\|_{1}=\left\|G_{\ell_{1}^{n+1}} F\right\|_{L_{1}\left(\ell_{1}^{n+1}\right)} \leq K\left(\ell_{1}^{n+1}\right)\|F\|_{L_{\infty}\left(\ell_{1}^{n+1}\right)}=K\left(\ell_{1}^{n+1}\right) \leq C^{\prime \prime}(1+\log n)^{1 / 2}$,

the last inequality being the well-known estimate for the (usual) $K$-convexity constant of $\ell_{1}^{m}$. Combining the last two inequalities yields the required majorant for $A$ and hence the second assertion of the Proposition.

Remark 5.6 Our final remark concerns possible use of the Rademacher projection in place of the Gaussian projection in section 4 (with almost the same outcome). Indeed, one may replace throughout the argument $\left(\mathbb{R}^{\mathbb{N}}, \mathcal{B}, \gamma_{\infty}\right)$ by $Z_{2}^{\mathbb{N}}=\{-1,1\}^{\mathbb{N}}$ equipped with the Haar measure, the Gaussian variables by the Rademachers, and the Hermite polynomials by the Walsh functions. In the symmetric case the norms of Gaussian and Rademacher projections are equivalent, more precisely $\|G\| \leq\|R\| \leq \frac{\pi}{2}\|G\|$ (see e.g., TomczakJaegermann (1989)). The proof of this fact does not use "strongly" the symmetry of the norm (only the boundedness of $\|x\| /\|-x\|$ is relevant). Moreover, for any finite sequence $\left(x_{i}\right)$ in $X$ we have

$$
\sqrt{\frac{2}{\pi}}\left\|\sum r_{i} x_{i}\right\|_{L_{2}(X)} \leq\left\|\sum g_{i} x_{i}\right\|_{L_{2}(X)} \leq C \sqrt{1+\log d_{X}}\left\|\sum r_{i} x_{i}\right\|_{L_{2}(X)}
$$

(see e.g., Tomczak-Jaegermann (1989), 4.2-4.4, or Bourgain and Milman (1987), Lemma 8.6, for the symmetric case); since Gaussian and Rademacher variables have symmetric distributions, this inequality holds also in the nonsymmetric case. Then we can define the functional $\varphi=\varphi_{R}$ for the Rademacher projection and obtain for it almost the same estimates as in the Gaussian case. More precisely, the Pisier's estimate $\|G\| \leq C\left(1+\log d_{X}\right)$ and Proposition 4.3 carry over (more or less) directly to the Rademacher case. Lemma 4.4 
can be obtained using Lemma 4.5 and a convolution with a Riesz measure (Pisier (1981), see also Bourgain and Milman (1987)). In Theorem 4.1 an additional logarithmic factor will appear, i.e.

$$
\operatorname{Ell}(K) \leq C n \varphi_{R}(K)\left(1+\log d_{X}\right),
$$

and so this approach leads to a slightly inferior final result.

\section{References}

Banaszczyk, W. (1996). Inequalities for convex bodies and polar reciprocal lattices in $\mathbb{R}^{n}$ II: Application of $K$-convexity. Discrete Comput. Geom. 16 305-311.

Barthe, F. (1998a). An extremal property of the mean width of the simplex, Math. Ann. 310 685-693.

Barthe, F. (1998b). On a reverse form of the Brascamp-Lieb inequality. Invent. Math. 134 335-361.

Benyamini, Y., Y. Gordon (1981). Random factorization of operators between Banach spaces. J. Analyse Math. 39 45-74.

Bourgain, J. (1984). On martingale transforms in finite dimensional lattices with an appendix on the K-convexity constant. Math. Nachr. 119 41-53.

Bourgain, J., V. D. Milman (1986). Distances between normed spaces, their subspaces and quotient spaces. Integral Equations Operator Theory 9 31-46.

Bourgain, J., V. D. Milman (1987). New volume ratio properties for convex symmetric bodies in $R^{n}$. Invent. Math. 88 319-340.

Brascamp, H. J., E. H. Lieb (1976). Best constants in Young's inequality, its converse and its generalization to more than three functions. Adv. Math. 20 151-173.

Figiel, T., N. Tomczak-Jaegermann (1979). Projections onto Hilbertian Subspaces of Banach spaces. Israel J. Math. 33 155-171.

Gritzmann, P., J. M. Wills (1993). Lattice points. P. M. Gruber and J. M. Wills eds., Handbook of Convex Geometry, North-Holland, Amsterdam, 765-797.

Grünbaum, B. (1963). Measures of symmetry for convex sets. Proc. Sympos. Pure Math. 7, Amer. Math. Soc., Providence, 233-270. 
John, F. (1948). Extremum problems with inequalities as subsidiary conditions. Courant Anniversary Volume, Interscience, New York, 187-204.

Kannan, R., L. Lovász (1988). Covering minima and lattice-point-free convex bodies. Ann. Math. 128 577-602.

Kantor, J.-M. (1998). On the width of lattice-free simplices. Preprint.

Khinchine, A. (1948). A quantitative formulation of Kronecker's theory of approximation. Izv. Acad. Nauk SSSR, Ser. Mat. 12 113-122 (in Russian).

Lenstra, H. W. (1983). Integer programming with a fixed number of variables. Math. of Oper. Res. 8 538-548.

Lewis, D. R. (1979). Ellipsoids defined by Banach ideal norms. Mathematika 26 no. $1,18-29$.

Lindenstrauss, J., V. D. Milman (1993). The local theory of normed spaces and its applications to convexity. P. M. Gruber and J. M. Wills eds., Handbook of Convex Geometry, North-Holland, Amsterdam , 1149-1220.

Litvak, A., N. Tomczak-Jaegermann (1998). Private communication.

Milman, V. D., G. Schechtman (1986). Asymptotic theory of finite dimensional normed spaces, Lecture Notes in Math. 1200, Springer Verlag, Berlin-New York.

Pełczyński, A. (1980). Geometry of finite-dimensional Banach spaces and operator ideals. E. H. Lacey, ed., Notes in Banach spaces, University of Texas Press, Austin, Texas, 81-181

Pisier, G. (1981). K-convexity. B.-L. Lin, ed., Proceeding of Research Workshop on Banach spaces and operator ideals, University of Iowa, Iowa City, Iowa, 139-151.

Pisier, G. (1986). Probabilistic methods in the geometry of Banach spaces. C.I.M.E. Varenna 1985. Lecture Notes in Math. 1206, Springer Verlag, Berlin-New York, 167-241.

Pisier, G. (1989). The Volume of Convex Bodies and Banach Space Geometry, Cambridge University Press, Cambridge.

Rudelson, M. (1998) Distances between non-symmetric convex bodies and the $M M^{*}$-estimate. Preprint. Available at http://xxx.lanl.gov/abs/math/9812010. 
Sebö, A. (1998). An Introduction to Empty Lattice Simplices. IPCO 7, Lecture Notes in Computer Science, Springer Verlag, to appear.

Talagrand, M. (1987). Regularity of Gaussian measures. Acta Math. 159 99-149.

Talata, I. (1997). Covering the lattice points of a convex body with affine subspaces. Intuitive geometry (Budapest, 1995), 429-440, Bolyai Soc. Math. Stud., 6, Janos Bolyai Math. Soc., Budapest, 1997.

Tomczak-Jaegermann, N. (1989)., Banach-Mazur distances and finite-dimensional operator ideals, Pitman Monographs and Surveys in Pure and Applied Mathematics, 38. Longman Scientific \& Technical, Harlow; copublished in the United States with John Wiley \& Sons, Inc., New York.

Faculty of Mathematics, Eódź University, 90-238 Eódź, Poland

E-mail: wbanasz@imul.uni.lodz.pl

Department of Mathematics Tel Aviv University Ramat Aviv, Tel-Aviv, Israel

Present address: Department of Mathematics, Technion, Haifa 32000, Israel

E-mail : alexandr@math.ualberta.ca

Equipe d'Analyse et de Mathématiques Appliquées, Université de Marne-la-Vallée, 5 blvd Descartes, 77454 Marne-la-Vallée, France

E-mail : pajor@math.univ-mlv.fr

Department of Mathematics, Case Western Reserve University, Cleveland, OH 441067058, U.S.A.

and

Université Pierre et Marie Curie, Equipe d'Analyse, Bte 186, 4, Place Jussieu, 75252

Paris, France

E-mail : szarek@ccr.jussieu.fr 